\title{
Enhancing the evaluation of pathogen transmission risk in a hospital by merging hand-hygiene compliance and contact data: a proof-of-concept study
}

\author{
Rossana Mastrandrea ${ }^{1}$, Alberto Soto-Aladro ${ }^{2,3}$, Philippe Brouqui ${ }^{2,3}$ and Alain Barrat ${ }^{1,4^{*}}$
}

\begin{abstract}
Background: Hand-hygiene compliance and contacts of health-care workers largely determine the potential paths of pathogen transmission in hospital wards. We explored how the combination of data collected by two automated infrastructures based on wearable sensors and recording (1) use of hydro-alcoholic solution and (2) contacts of health-care workers provide an enhanced view of the risk of transmission events in the ward.

Methods: We perform a proof-of-concept observational study. Detailed data on contact patterns and hand-hygiene compliance of health-care workers were collected by wearable sensors over 12 days in an infectious disease unit of a hospital in Marseilles, France.

Results: 10,837 contact events among 10 doctors, 4 nurses, 4 nurses' aids and 4 housekeeping staff were recorded during the study. Most contacts took place among medical doctors. Aggregate contact durations were highly heterogeneous and the resulting contact network was highly structured. 510 visits of health-care workers to patients' rooms were recorded, with a low rate of hand-hygiene compliance. Both data sets were used to construct histories and statistics of contacts informed by the use of hydro-alcoholic solution, or lack thereof, of the involved health-care workers.

Conclusions: Hand-hygiene compliance data strongly enrich the information concerning contacts among healthcare workers, by assigning a 'safe' or 'at-risk' value to each contact. The global contact network can thus be divided into 'at-risk' and 'safe' contact networks. The combined data could be of high relevance for outbreak investigation and to inform data-driven models of nosocomial disease spread.
\end{abstract}

Keywords: Hand-hygiene compliance, Contact networks, Potential pathogen transmission

\section{Background}

Hospital Acquired Infections (HAI) represent a major risk for patient safety and health, leading to prolonged stay and increased mortality [1-3], and has high economic costs. The dynamics of pathogen transmission in hospital wards involves at least two components linked to the behavior of healthcare workers (HCWs): compliance to hand-hygiene protocols $[4,5]$ and contacts between

\footnotetext{
*Correspondence: alain.barrat@cpt.univ-mrs.fr

${ }^{1}$ Aix Marseille Université, Université de Toulon, CNRS, CPT, UMR 7332,

13288 Marseille Cedex 9, France

Full list of author information is available at the end of the article
}

HCWs that might lead to transmission events $[6,7]$. In order to better understand such dynamics and ultimately develop prevention and containment measures, recent studies have concerned each of these two components.

On the one hand, detailed investigations of handhygiene compliance (HHC) of HCWs have been performed using techniques ranging from direct observation, still considered as a gold standard but costly, to sensors monitoring the use of hydro-alcoholic solution (HAS) by HCWs [8-10].

On the other hand, the recent development of unobstrusive wearable sensors has also made possible the collection of data describing the contact patterns of 
individuals with high spatial and temporal resolutions in various contexts [11-14], including hospital wards [15-19]. Moreover, the combination of contact data with microbiological data has been shown to provide evidence for the occurrence of transmission events of pathogens upon close proximity of individuals $[18,19]$.

Contact data and hand-hygiene compliance data have however, to our knowledge, not been studied in combination, although numerical studies using agent-based models of hospital wards have clearly highlighted that both aspects play an important role in the potential spread of HAI outbreaks [6,7]. Here, we perform a first step in this direction through a proof-of-concept study in which we explore the merging of data coming from two datacollection infrastructures based on wearable sensors: MediHandTrace (MHT) [9], which monitors the use of HAS by HCWs, and the SocioPatterns infrastructure, which records the close face-to-face proximity of individuals. We show how combining such data provides an enhanced view of the risk of transmission events in the ward: indeed, the temporal network of contacts between HCWs and of visits of HCWs to patients' rooms results enriched by the information about hand-hygiene compliance of the individuals involved in each contact event. Such combined data could be of high relevance for outbreak investigation and to inform data-driven models of nosocomial disease spread.

\section{Methods}

\section{Setting and data collection}

Data were collected from Monday, July 7, 2014 to Friday, July 18, 2014 without interruption, i.e., 24 h per day, in the Infectious Disease Unit of the Hôpital Nord in Marseilles, France. The study involved 7 rooms with patients and 22 healthcare workers (HCWs), categorized into 4 classes according to their role in the ward: 10 medical doctors (MED), 4 nurses (NUR), 4 nurses' aids (NURA) and 4 housekeeping staff (HKS). HCWs were present in the ward in shifts of $8 \mathrm{~h}$.

For each day, each room was charaterized by a specific type of isolation requirement: "Standard" (no specific isolation procedure), "Contact", "Respiratory" and "Clostridium". For rooms in the "Clostridium" category, strong hygiene measures are required: HCWs are to wear gloves and special clothes before entering the room, downing gloves and clothes in the room before exit. Here, hand-hygiene compliance is considered as particularly important, especially after contact with the patient after removing gloves.

\section{Contact data}

We recorded close proximity face-to-face interactions ("contacts") among HCWs in the ward, using the proximity-sensing platform developed by the SocioPatterns collaboration [11] and described in detail in [12, 16]. The SocioPatterns platform elements are open and commercially available from e.g. the Bitmanufaktur company (http://www.openbeacon.org). Participating individuals were asked to wear unobstrusive badges on their chests, equipped with radio-frequency identification sensors that exchange ultra-low power radio data packets when close enough (1-1.5 m). The sensors were powered by batteries that ensured a lifetime of about 12 days, so that no battery exchange was necessary during the data collection (a new version of the sensors, currently under development, will ensure continuous functioning of the sensors for several months without change of battery). The system was tuned so that it detected and recorded close-range encounters during which a communicable disease infection could be transmitted, for example, by cough, sneeze or hand contact. The temporal resolution was set to $20 \mathrm{~s}$, and the information on face-to-face proximity events detected by the wearable sensors was relayed to radio receivers installed throughout the hospital ward in a way to ensure redundancy (most events were registered by more than one radio receiver) and avoid data losses. A sensor was also placed in the toilet just above the washbasin and close to the HAS dispenser. Contacts registered between this sensor and wearable sensors of HCWs were interpreted as a signal of handhygiene compliance.

\section{MediHandTrace (MHT) data}

Seven patient rooms, among the 16 in the ward, are currently equipped with the MediHandTrace infrastructure (see http://www.medihandtrace.com), namely floor-embedded antennas able to read passive RFID (radio-frequency identification) tags inserted in the shoes of 11 of the $22 \mathrm{HCWs}$ (4 medical doctors, 2 nurses, 3 housekeeping staff and 2 nurses' aids). Only 7 rooms have up to now been equipped with such antennas, as the system is still in an experimental phase. Moreover, the $11 \mathrm{HCWs}$ who did not wear such shoes were medical students, who were in the ward only for few weeks, while the MHT surveillance system is currently permanently active and measurements focus on the permanent staff.

For each room, the antennas are located around the HAS dispensers (one outside and one inside the room), at the room door and around the patient's bed [9]. The antennas are connected to a reader, which transmits the information to a central server through an Ethernet connection. For each HCW equipped with a sensor, information about the presence in a room is detected by the antennas located at the room door, use of HAS is inferred by the detection of the sensors close to the 
HAS dispensers, and the vicinity of the $\mathrm{HCW}$ to a patient is detected by the antenna located around the patient's bed. These events are transmitted in real-time to a centralized server. The MHT infrastructure has been validated against direct observation in a previous publication [9].

\section{Data analysis}

We first analyzed each data set separately. For both data sets, we moreover exclude in the analysis the night periods, as almost no events were registered during these periods, and keep only the time intervals from 6:00 AM to 8:00 PM.

We studied the contacts between HCWs by focusing on contact matrices giving the number and durations of contacts between categories of HCWs and on the temporal evolution of these quantities during the day. We moreover built the daily and global networks of contacts between HCWs: in such networks, nodes represent HCWs and a link is drawn between two nodes if the corresponding individuals have met at least once in the period considered; each link is weighted by the total duration of the contacts between the concerned HCWs during the aggregation period.

We analyzed the MHT data by measuring, for each HCW category, the average number and duration of visits in patient rooms, as measured by the antennas located at each room entrance, as a function of the hour of the day. We moreover considered the (daily and global) bipartite network of visits between HCWs and rooms: nodes represent either HCWs or rooms, and a link is drawn between a HCW-node and a roomnode if the corresponding $\mathrm{HCW}$ visited the room at least once; each link is weighted by the total duration of the visit(s) and can moreover be characterized by the $\mathrm{HHC}$ of the HCW during the visit. We also investigated the overall and individual $\mathrm{HHC}$ of $\mathrm{HCWs}$.

Merging the two data sets yields a temporally resolved contact network between HCWs in which each contact is enriched by the information about the $\mathrm{HHC}$ of the involved HCWs before the contact. We considered specific examples of HCWs contact histories and explored how HCWs' contacts can be divided into 'at-risk' and 'safe' categories. We built the corresponding daily and global aggregated contact networks.

\section{Ethics and privacy}

An oral and written consent to participate in the study was obtained from the HCWs. In order to ensure anonymity of the data, a random number was attributed to each participant when entered in the database. This study was declared to the French Commission on Individual Data Protection and Public Liberties (CNIL).

\section{Results and discussion Contact data}

A total of 10,837 contact events was recorded during the study, with a cumulative duration equal to $453,960 \mathrm{~s}$ ( $7566 \mathrm{~min}$ or $126 \mathrm{~h})$. The durations of single contacts and the aggregated durations of contacts between HCWs were highly heterogeneous (see Additional file 1): most contacts had very short durations, but some were very long, with no characteristic interaction time-scale. Most contact activity occurred within the medical doctors category on the one hand and between the nurses' aids and the housekeeping staff on the other hand (Fig. 1a). We show in the Additional file 1 that daily contact matrices exhibit the same structure as the global one. Contacts occurred mostly between 10:00 and 14:00 for medical doctors and nurses, while contacts between nurses' aids and housekeeping staff spread more evenly along the course of the day (Fig. 1b). More detailed insight into the contact patterns is provided by the contact networks of HCWs: the global aggregated network is shown in Fig. 2a) using a circular layout with HCWs grouped by category (daily networks are shown in the Additional file 1). Overall, medical doctors form a well-connected group connected to the rest of the network mostly through contacts with a couple of nurses. Figure $2 \mathrm{~b}$ ) provides network statistics for each $\mathrm{HCW}$, namely, degree (i.e., the number of other HCWs with whom a given $\mathrm{HCW}$ has had contacts) and strength (i.e., cumulative contact time of each $\mathrm{HCW}$ ). While the degrees do not exhibit very strong variations (as in other studies $[12,16$, 17]), different HCWs can have very different cumulative contact times. In particular, only 5 (resp. 10) HCWs out of 22 account for $44 \%$ (resp. $72 \%$ ) of the total contact time of all HCWs. Figure 2c finally shows the possibility to focus on the contact history of a specific HCW and to capture at a glance how distinct contact patterns occured at specific periods: for instance, HCW 113 (housekeeping staff) met repeatedly HCW 127 (nurses' aid) in different days, and met HCW 67 (medical doctor) for a long time but just on 1 day.

\section{Visits to patients and hand-hygiene compliance}

During the data collection, 510 visits of $\mathrm{HCWs}$ to patients were registered with an average duration of $247 \mathrm{~s}$ ( $\sim \mathrm{min})$. Figure $3 \mathrm{a}$ shows that most visits occured in the first part of the day, with a peak before noon. Most visits were performed by nurses' aids and housekeeping staff, while medical doctors performed only few visits, always around 1:00 PM. Figure 3b displays the bipartite network involving HCWs and rooms, aggregated over all the study (daily networks are shown in the Additional file 1): each link indicates that a HCW has visited a room at least once, and its width indicates the total (cumulated) 

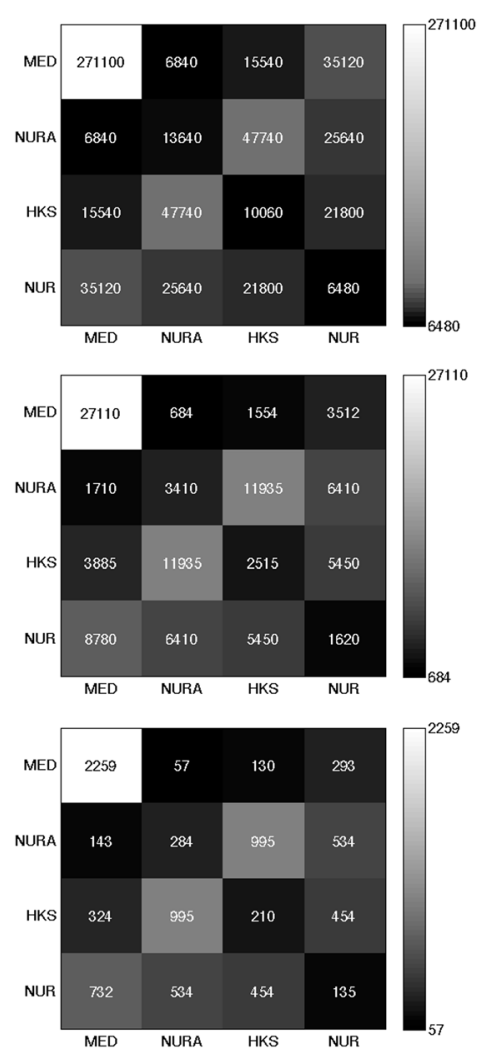

(a)
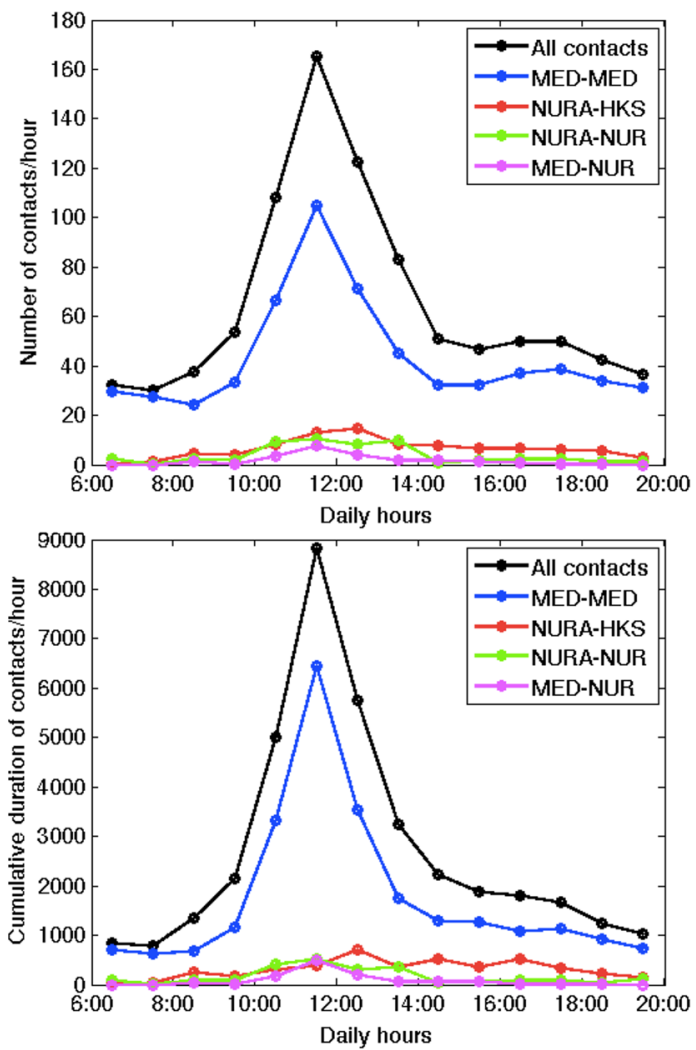

(b)

Fig. 1 Contacts between health-care workers. a Contact matrices: the top matrix gives at row $X$ and column $Y$ the aggregate duration of contacts between HCWs of category $X$ and HCWs of category $Y$; in the second matrix, each $(X, Y)$ entry is normalized by the number of HCWs in category $X$, giving thus the average time spent by a HCW of category $X$ with all HCWs of category $Y$; in the bottom matrix, each entry is moreover normalized by the duration (in days) of the data collection. b Number (top) and aggregate duration (bottom) of contacts as a function of the hour of the day, averaged over all the days of data collection. Data are shown for all contacts and for some specific categories. MED medical doctors; NUR nurses; NURA nurses' aids; HKS housekeeping staff

durations of the visits. The network is dense, with 63 links out of the 77 possible ( $82 \%)$. The degree of HCW nodes (number of different rooms visited) varies between 2 and 7, while the degree of room nodes (number of distinct HCWs who have visited the room) goes from 7 to 11. The figure highlights the weak participation of medical doctors to visits and, on the opposite, the large cumulated time spent by some HCWs in some rooms.

For each visit registered by the antennas located at each room entrance, the MHT infrastructure gave moreover information on the occurrence of an event of proximity with the patient ("contact"), detected by the antennas located around the beds, and on the handhygiene compliance of the $\mathrm{HCW}$, as inferred by the proximity events registered by the antennas located around the HAS dispensers. For each HCW, we can thus compute the compliance by the fraction of detected visits including proximity with the patient for which proximity with the HAS dispensers was also detected.
We note that this procedure has been validated against direct observation in Ref [9]. Figure 3c provides the resulting statistics on the use of HAS of each $\mathrm{HCW}$ before and/or after contact with a patient (further statistics can be found in the SI). Consistently with previous studies $[8,9,21,22]$, completely correct use of HAS was rare $(13 \%)$, while many visits (49\%) occurred with no HAS use at all. The remaining visits were characterized by a partial use, mostly after contact with the patient: $30 \%$ (resp. $8 \%$ ) with HAS use after (resp. before) the contact. Note that we do not take into account here the potential use of the bathroom as an alternative way to wash hands after visit to a room, as the MHT infrastructure by itself does not detect such events. We did not observe any clear trend of the hand-hygiene compliance as a function of the number or durations of visits, nor depending on the type of isolation of the room (data in Additional file 1). Overall, hand-hygiene compliance was scarce on average for all HCW categories, but 


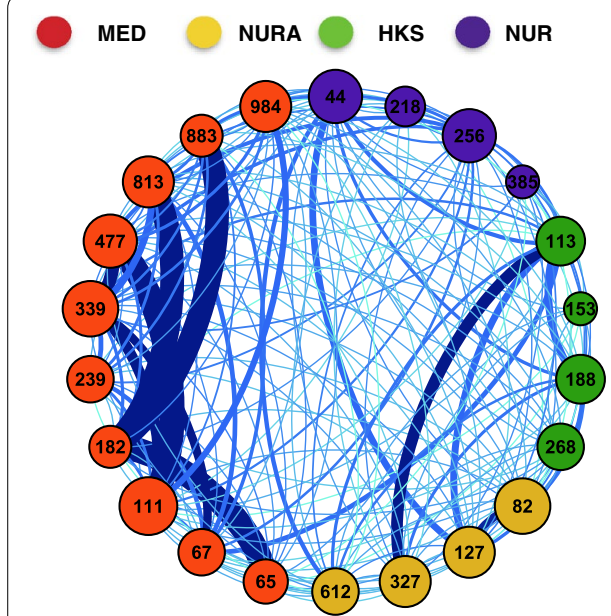

(a)

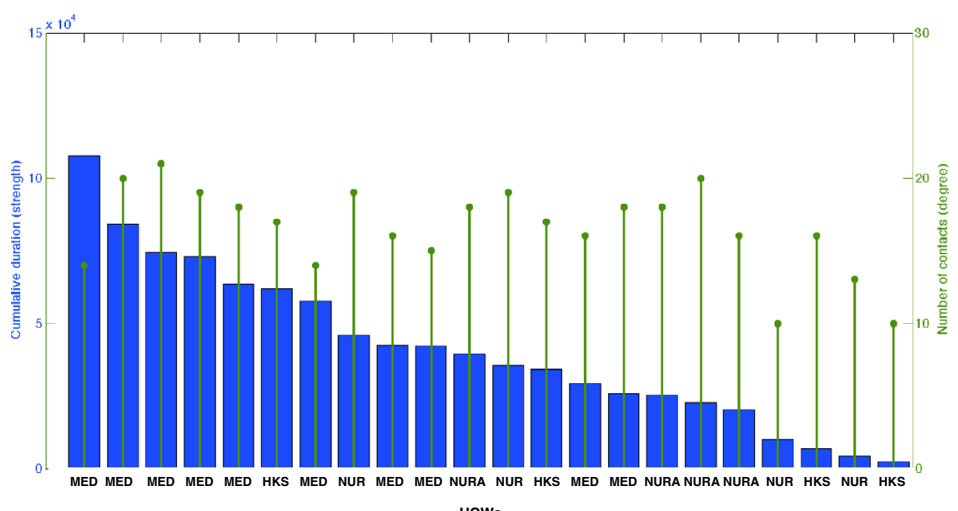

(b)
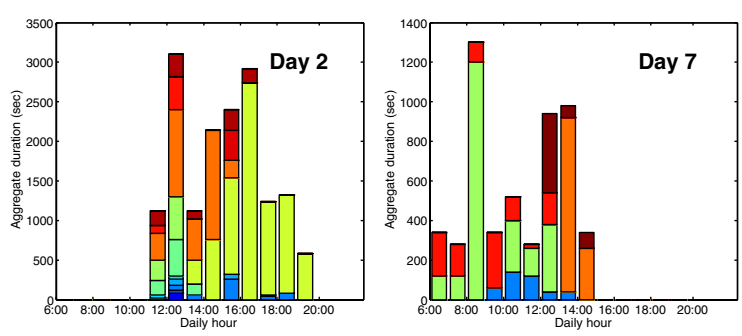
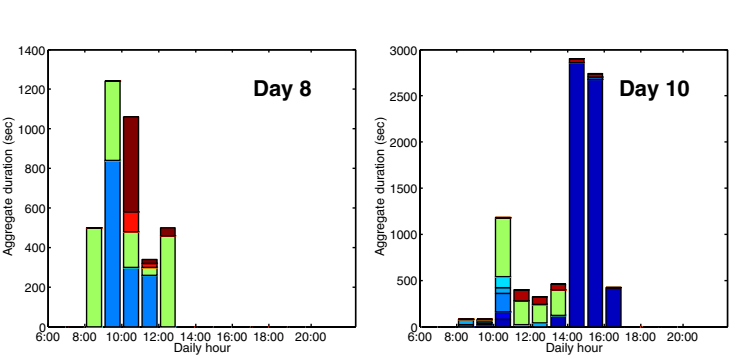

Other HCWs

(c)

Fig. 2 Contact network of health-care workers. a Visualization of the contact network of HCWs, aggregated over the whole data collection. Each node represents a HCW (the number stands for the RFID number, the color for the category as indicated in the color code). A link between two nodes exists if at least one contact between the corresponding HCWs was detected, and the link thickness gives the aggregate duration of contacts. The size of each node is given by its degree (number of links). $\mathbf{b}$ Cumulative duration (blue bars) of the contacts of each HCW, and number (green) of distinct other HCWs met by each HCW, during the whole data collection. These quantities correspond respectively to the strength and the degree of each node in the network of a. c Contact patterns of a specific HCW along several days. Each bar gives, for each hour of the day, the cumulative duration of contacts between HCW 113 (housekeeping staff) and other HCWs identified each by a specific color. MED medical doctors, NUR nurses, NURA nurses'aids; HKS housekeeping staff

important differences were observed between HCWs (see Additional file 1).

\section{Merging the two datasets}

We merged the data sets describing HCWs' visits to patients' rooms, their hand-hygiene compliance and their contacts, obtaining a detailed time-resolved picture of daily paths and contacts of HCWs with associated risks of hand transmission of pathogens due to the lack of hand-hygiene. The merged data concerns 9 HCWs: 2 medical doctors, 2 nurses, 2 nurses aids, 3 housekeeping staff. Figure $4 \mathrm{a}$ illustrates the richness of the combined data by showing visits, hand-hygiene and contacts of a specific HCW (113): contacts of 113 occurring between two room visits are shown together, with a bar height proportional to the contacts' durations, and the colored dot on top of the bar indicates if 113 used HAS or not after contact with a patient and before contact with the other HCWs. Information about the use of HAS by the HCW with whom 113 had a contact is also included.

We now focus on the contacts between two HCWs in which at least one of them participated to the MHT data collection, and such that the encounter occurred after a contact with a patient. This corresponds to 2593 contact events ( $24 \%$ of all contact events) of total duration $132,420 \mathrm{~s}$ ( $29 \%$ of the aggregated duration of all contacts) and concerning 92 of the 182 links between HCWs (51 \%). To each such contact, we can associate a risk of hand transmission of pathogens, depending on the HCW's hand-hygiene compliance (note that we do not take into account here the contacts occurring at the start of the HCWs shifts, before they have any contact 


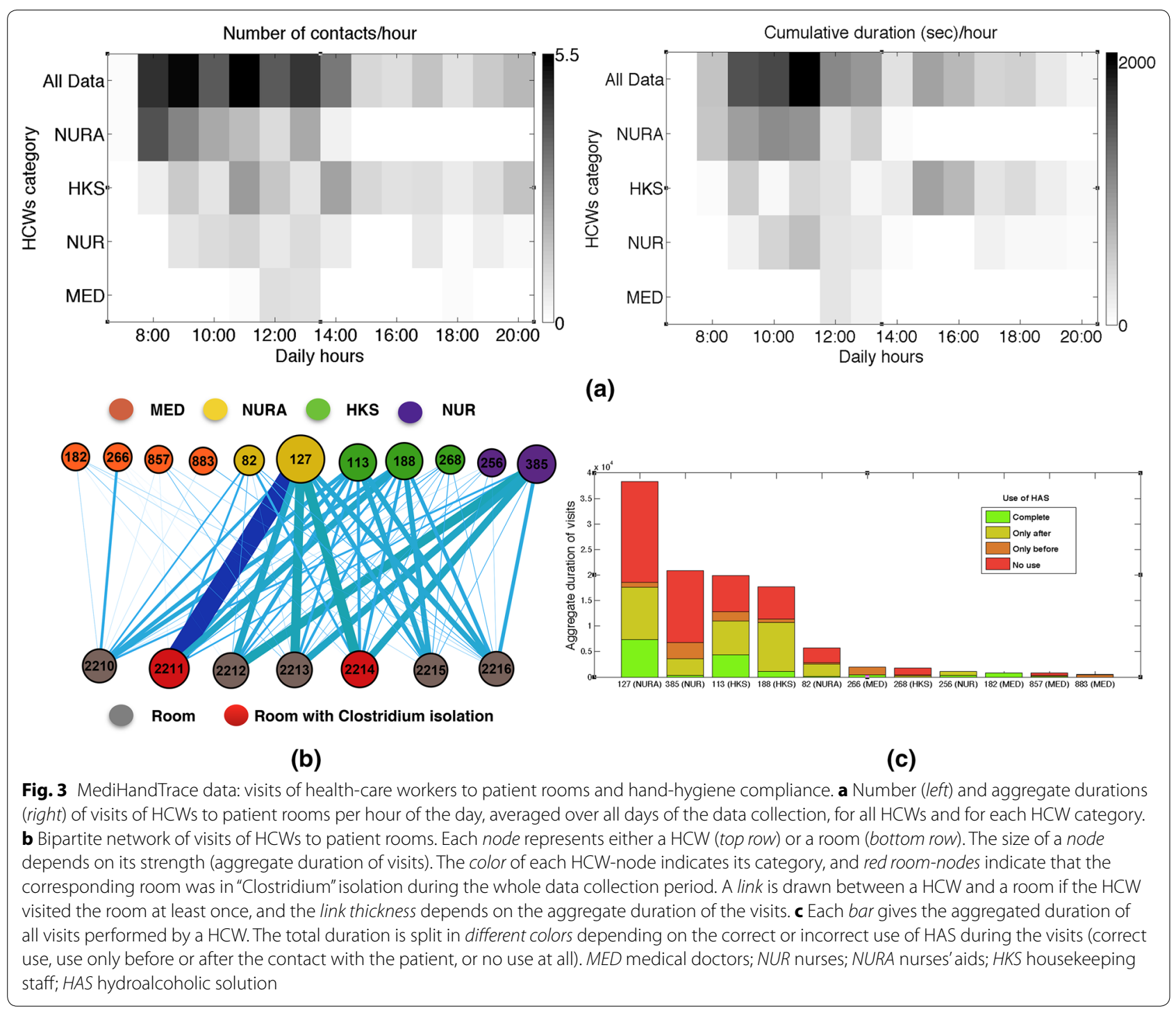

with patients). Here, hand-hygiene compliance after contact with a patient can correspond either to the use of HAS in the same room in which contact with the patient occurred, as measured by MHT, or proximity to the bathroom washbasin as detected by the SocioPatterns infrastructure. Figure 4 reports the corresponding statistics at the level of pairs of HCWs (b) and of individual HCWs (c). Each bar gives the total duration of the measured contacts between a pair of HCWs (resp. of all contacts of each $\mathrm{HCW}$ ), and is divided into durations of 'at-risk' and 'safe' contacts, according to the use of HAS by the HCWs before the contact. The average aggregate duration of 'safe' contacts was larger than the one of 'at-risk' contacts ( 286 vs. 190 s), but large variations, with both short and long contacts, were observed in each case. We did not observe any significant correlation between total contact durations and hand-hygiene compliance of HCWs. Overall, $7 \mathrm{HCWs}$ out of 9 had contacts with other HCWs after contact with a patient and lack of HAS use.

Figure 5 finally illustrates how networks of contacts between HCWs can be enriched by the HHC data and divided into two networks corresponding to 'at-risk' and 'safe' contacts. We show in Fig. 5 the global contact network and two daily networks, each one split according to this criterion. In each layout, nodes are placed in the same location, and the link thickness corresponds to the aggregated duration of the contacts between the linked nodes. 'At-risk' and 'safe' contact networks show different patterns, with some links present in both while others are observed only in one. In particular, only the 'at-risk' network should be considered in a hypothetical scenario of hand transmission of pathogens. Risk assessment analysis 


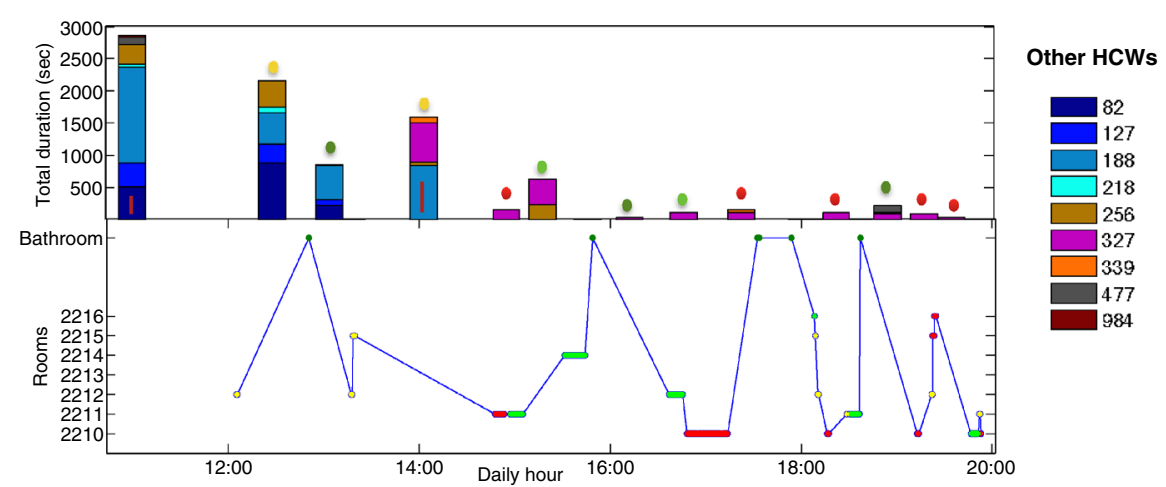

(a)

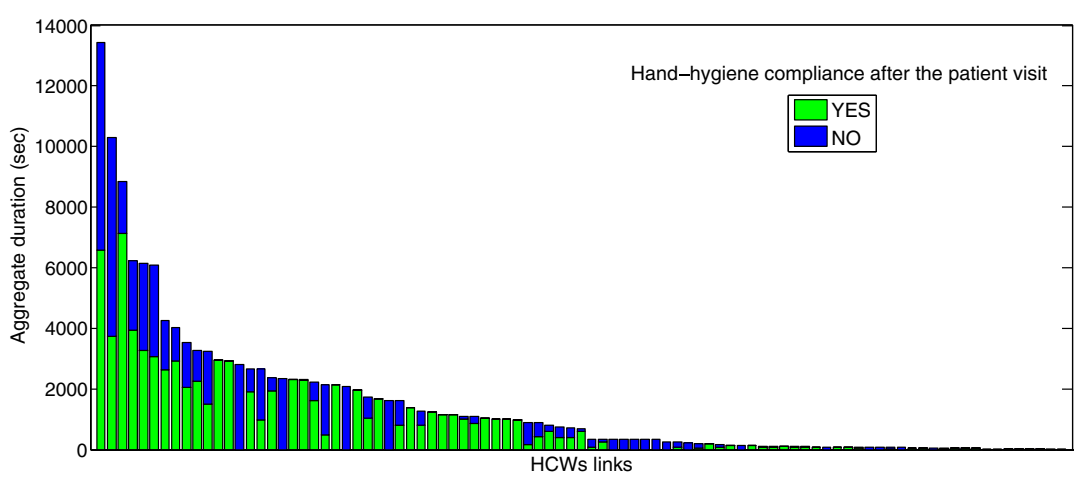

(b)

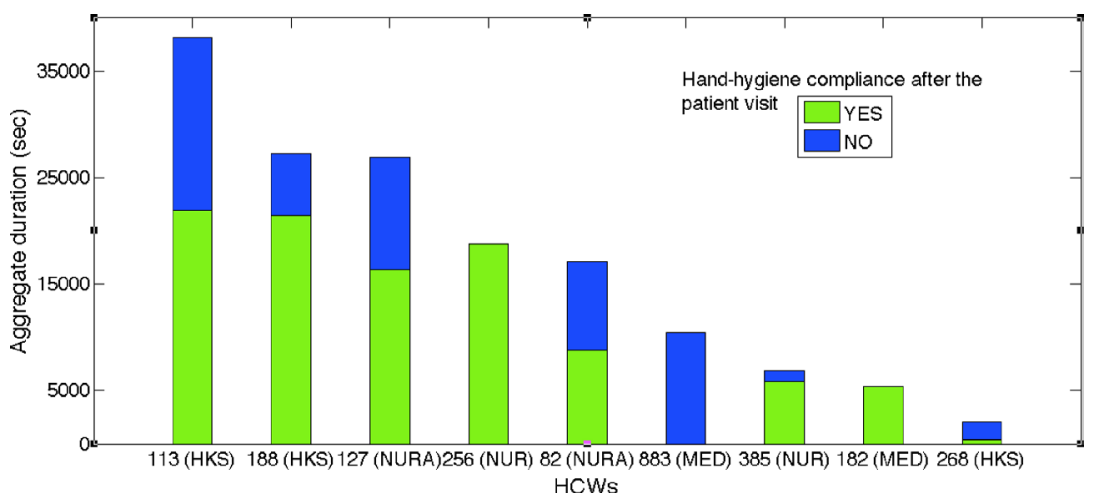

(c)

Fig. 4 HCW contacts and hand-hygiene compliance. a Contact patterns of a single HCW (113, ASH) during a specific day, informed by handhygiene compliance data. Bottom each dot represents a visit to a room, and its color depends on the use of HAS, or lack thereof, after a contact with the patient in the room (red no use; light green use; dark green bathroom; yellow no contact with the patient). Top each bar gives the duration of a contact of HCW 113 with another HCW, identified by a specific color. The colored dot indicates if HCW 113 had used HAS in the previous visit (same color code as in the bottom plot). The red line in some colored bars indicate that the HCW in contact with HCW 113 had previously visited a patient room with successive lack of HAS use. $\mathbf{b}$ Bar chart giving the aggregate duration of the contacts of each pair of HCWs. Only encounters between HCWs of which at least one wore a MediHandTrace (MHT) tag monitoring hand-hygiene compliance, and had a contact with a patient before the encounter, are considered. Each duration is divided into two parts corresponding to different hand-hygiene compliance (green correct use of HAS of the HCWs monitored by the MHT infrastructure before the contact; blue no use of HAS after contact with the patient and before the contact for at least one of the two HCWs involved). c Bar chart giving, for each HCW, the aggregate duration of his/her contacts with other HCWs after contact with a patient. Each aggregate duration is divided into two parts corresponding to different hand-hygiene compliance (green correct use of HAS after contact with the patient and before the contact with the other HCW; blue no use of HAS). MED medical doctors; NUR nurses; NURA nurses' aids; HKS housekeeping staff; HAS hydroalcoholic solution 


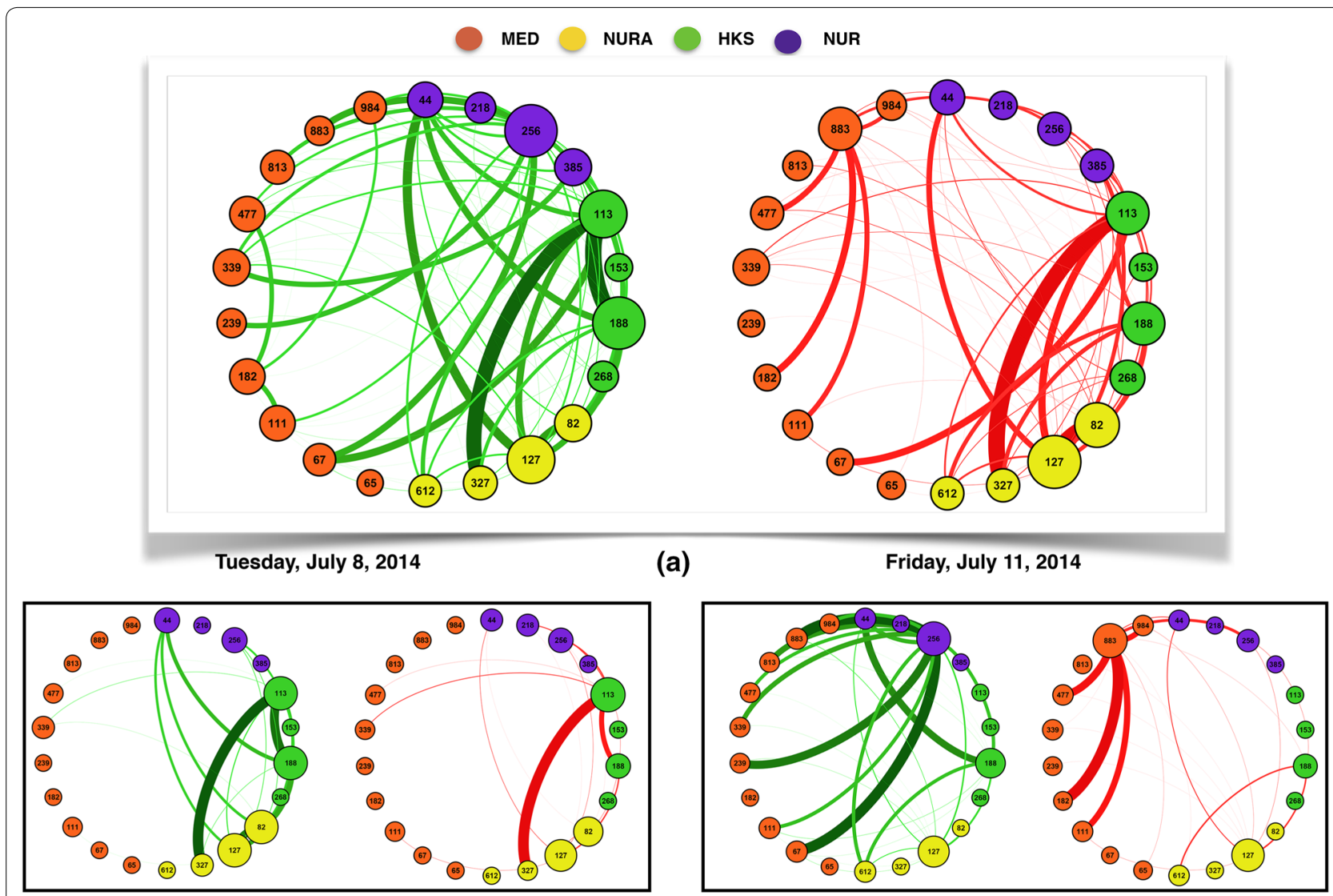

(b)

Fig. 5 Networks of HCWs contacts informed by the hand-hygiene compliance data. In each network, similarly to Fig. 2a, nodes represent HCWs, with a different color according to each HCW's category, and a link between two nodes indicates that the corresponding HCWs have been in contact at least once. Here only contacts for which at least one of the HCWs was monitored by the MediHandTrace (MHT) infrastructure, and occurring after at least one of the HCWs involved had a contact with a patient, are taken into account. We create two contact networks by integrating the information about hand-hygiene compliance of HCWs: the network of green links corresponds to contacts in which HCWs monitored by MHT have used HAS before the contact; the network of red links corresponds to contacts occurring after at least one of the HCWs had a contact with a patient and did not use HAS afterwards. In each network, the links thickness depends on the aggregate duration of contacts. a Networks aggregated over the whole data collection; b two examples of daily contact networks. MED medical doctors; NUR nurses; NURA nurses' aids; HKS housekeeping staff, HAS hydroalcoholic solution

should thus focus on this network and its characteristics. We also emphasize that Fig. 5 gives only static representations of a temporal network, in which each link represents an aggregation of several contact events. The whole data set includes the temporality of contacts together with their risk status in terms of hand-hygiene of the involved HCWs.

\section{Discussion}

The objective of this work was to show how merging data concerning contacts and hand-hygiene compliance of health-care workers in a hospital ward can provide an enriched picture of transmission risks in such contexts. Previous data collection and data-driven studies focused indeed typically on only one of these aspects. For each separate data set, we obtained results consistent with previous works. For instance, the duration of contacts was heterogeneous, with the coexistence of contacts of very different durations and the emergence of "supercontactors" who account for a large fraction of all observed contacts $[12,14,16,17]$. In terms of contagion risk, this implies that different contacts can correspond to very different probabilities of transmitting a pathogen. The contact network was moreover strongly structured as in other studies [12, 14, 16, 17]. These results confirm the need to go beyond the contact matrix description that only accounts for averaged contact durations between different categories of workers [20] and to include 


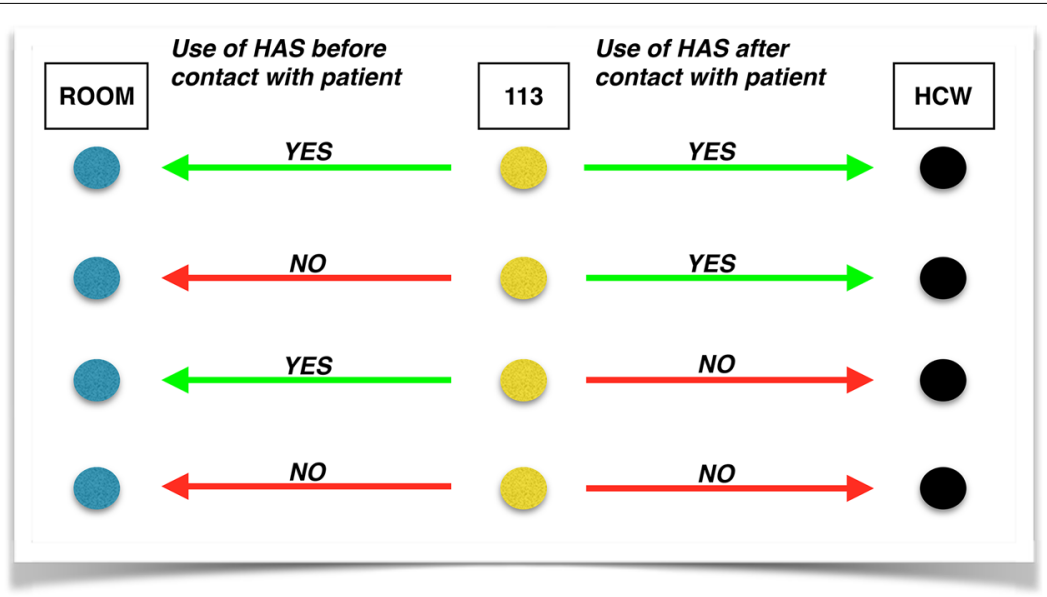

(a)

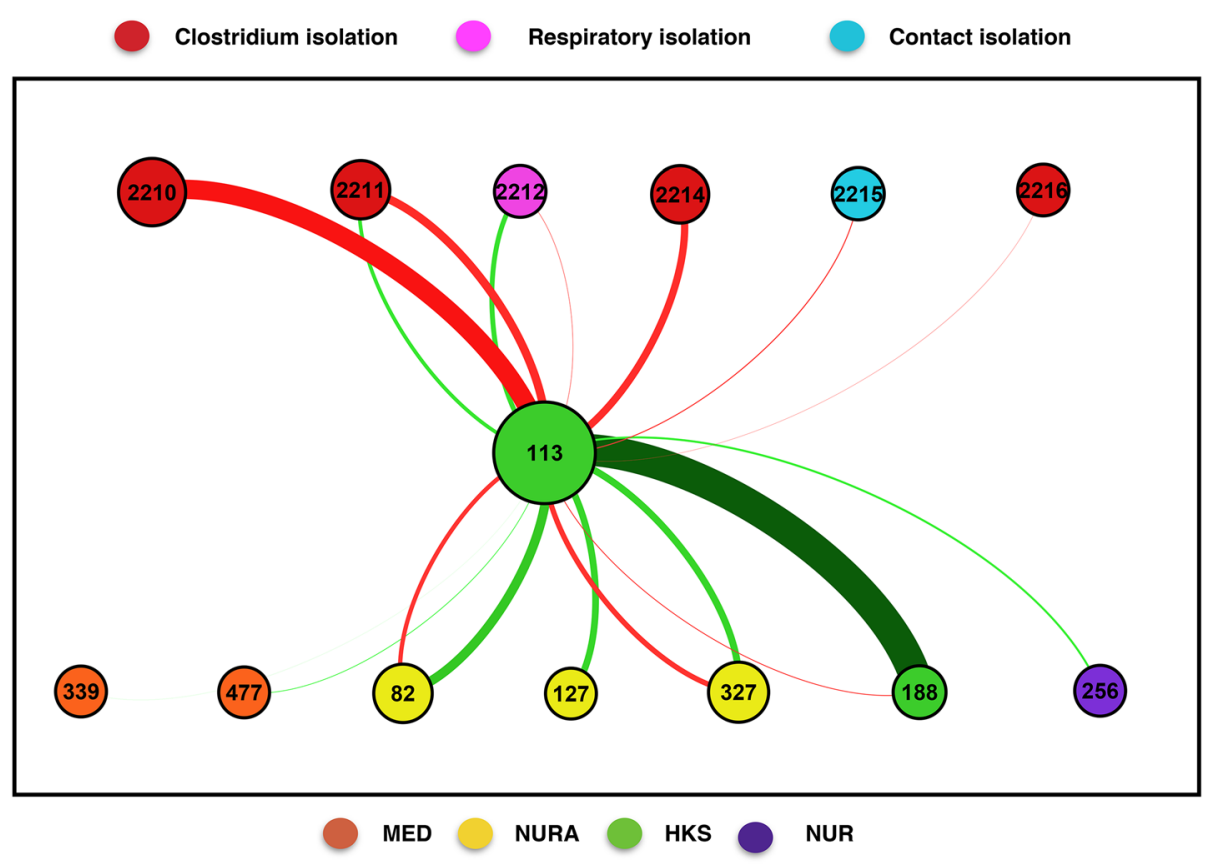

(b)

Fig. 6 Illustration of the complexity of the network including HCWs' contacts and rooms'visits. a An example of the different types of contacts involving a specific healthcare worker after a visit to a patient. The hand-hygiene compliance can affect both HCWs and patients, with four possible scenarios according to the use of HAS before and after the visit, as illustrated in the figure: the first line corresponds to HCW 113 using HAS before contact with a patient (green arrow from 113 towards the room) and also after the contact with the patient and before the contact with the other HCW (green arrow towards the other HCW); the second line corresponds to HCW 113 having a contact with the patient in the room without previous use of HAS (hence a risk of transmission to the patient red arrow) but using HAS after the contact with the patient and before contact with the other HCW. Lines 3 and 4 correspond to the scenarios of lines 1 and 2 respectively, but without use of HAS after the contact with the patient. $\mathbf{b}$ Network of all the contacts between a specific HCW (113), patients (indicated by their room number) and other HCWs during a specific day. A link between two nodes exists if the contact was observed at least once in the day; green and red links correspond to the use (or lack thereof) of HAS before the contact with the patient or between contact with patient and contact with HCW; the thickness of each link stands for the cumulative duration of the corresponding contacts. This figure integrates the information about at-risk and safe contacts of Fig. $5 \mathbf{a}$ with the information about the use of HAS before the visit to the patient (i.e., the risk associated to the encounters HCW-patient). MED medical doctors; NUR nurses; NURA nurses' aids; HKS housekeeping staff, HAS hydroalcoholic solution 
information about contact network structure and heterogeneity of contacts duration in data-driven models of disease spreading or in outbreak investigations.

Differently from other studies, most contacts were observed among medical doctors and between nurses'aids and housekeeping staff, due to a specific organization of work in the ward with pairs of one AS and one ASH working together.

With regards to hand-hygiene compliance, results were in line with other studies $[8,9,21,22]$, with only $13 \%$ of visits of HCWs to room patients including a correct use of HAS (both before and after contact with the patient). The widely spread lack of hand-hygiene compliance emphasizes the interest of merging the two data sets: on the one hand, the risk of pathogen transmission during a contact between HCWs can strongly depend on the occurrence of visits to patients and of the corresponding use of HAS (or lack thereof) after contact with a patient and before the HCW-HCW contact; on the other hand, it shows how lack of HAS use should not be considered as an isolated phenomenon but can have far-reaching consequences as pathogens transmitted from a patient to a HCW could then easily be transmitted through the HCW contact network and spread throughout the ward. It emphasizes how each contact with a patient and each hand-washing event are not isolated events but part of a complex network of events.

We have illustrated how simple visualizations of HCWs visits and contacts can be obtained by such data and could be of use in outbreak investigation. We have also shown how the network analysis of HCWs' contacts is enriched by taking into account hand-hygiene data. The network could be made even richer by combining the networks of HCWs' contacts and visits, and assigning a risk to each link according to the use of HAS. Note that in the present proof-of-concept study, we have focused on use of HAS after contact with a patient, which determines the risk of HCW-HCW contacts, while such an enriched network could also encode information on the use of HAS before the contact with a patient in the links between room-nodes and HCWnodes, as illustrated in Fig. 6 . This network thus includes information not only on the risk of pathogen transmission between HCWs but also from HCWs to patients and provides a picture of the possible paths of pathogen transmission in the ward that would be more complete than usual descriptions based only on contact patterns (due to the complexity of the representation, we show in Fig. 6 only the part of the network around a specific HCW.) We finally note that other potential ways of transmission of pathogens between HCWs and HCWs and patients exist in a ward, such as through clothing and equipment. A perfect, but obviously idealized, surveillance system would therefore be able to monitor also their correct use and disposal.

\section{Conclusion}

Hand-hygiene compliance data strongly enrich the information concerning contacts among health-care workers: indeed, each contact can be assigning a 'safe' or 'at-risk', yielding two distinct 'at-risk' and 'safe' contact networks. The resulting enriched temporal contact network could be of high relevance for outbreak investigation and to inform data-driven models of nosocomial disease spread.

Further work includes repeated data collections and analysis in order to test the robustness of the statistical patterns observed, as well as the design of data-driven models informed by the collected data to simulate the potential spread of pathogens and identify efficient containment measures in case of an outbreak. The results presented here will also be used to provide feedback to HCWs and attract their attention on the complex interplay of contacts and hand-washing events and how lack of hand-hygiene compliance can potentially lead to consequences involving the whole network of HCWs. As the question of how to increase hand-hygiene compliance among HCWs is still wide open [21], the effect of such feedback could then be measured in a new data collection campaign.

\section{Additional file}

Additional file 1. Additional analysis including in particular the daily contact patterns of healthcare workers and a detailed investigation of their hand-hygiene behaviour.

\section{Abbreviations}

HCW: health-care worker; HHC: hand-hygiene compliance; HAS: hydroalcoholic solution; MHT: MediHandTrace; MED: medical doctors; NUR: nurses; NURA: nurses' aids; HKS: housekeeping staff.

\section{Authors' contributions}

$A B$ and $P B$ conceived and designed the study. $A B, P B, A S, R M$ deployed the infrastructures and collected the data. RM analyzed the data, performed the statistical analysis, created the figures and wrote the first draft of the manuscript. AB, PB and RM wrote the final version of the manuscript. All authors read and approved the final manuscript.

\section{Author details}

Aix Marseille Université, Université de Toulon, CNRS, CPT, UMR 7332

13288 Marseille Cedex 9, France. ${ }^{2}$ Aix Marseille University, IHU Méditerranée Infection, URMITE, UM63, CNRS 7278 IRD 198, Inserm 1095, Marseille, France. ${ }^{3}$ Infectious Disease Unit CHU Nord, Institut Hospitalo-Universitaire Méditerranée Infection, Marseille, France. ${ }^{4}$ Data Science Laboratory, ISI Foundation, Turin, Italy

\section{Acknowledgements}

We thank the SocioPatterns collaboration for providing access to the SocioPatterns sensing platform that was used in collecting the contact data. We are grateful to the hospital staff who volunteered to participate in the data collection. This work has been carried out thanks to the support of the A*MIDEX project (no ANR-11-IDEX-0001-02) funded by the «Investissements d'Avenir » French Government program, managed by the French National 
Research Agency (ANR). A.B. is partially supported by the French ANR project HarMS-flu (ANR-12-MONU-0018) and by the EU FET project Multiplex 317532. The authors have no conflict of interest. The funding bodies had no role in the design of the study nor in the data analysis and interpretation.

\section{Compliance with ethical guidelines}

\section{Competing interests}

The authors declare that they have no competing interests.

Received: 4 June 2015 Accepted: 31 August 2015

Published online: 10 September 2015

\section{References}

1. Cosgrove SE. The relationship between antimicrobial resistance and patient outcomes: mortality, length of hospital stay, and health care costs. Clin Infect Dis. 2006;42((Suppl. 2)):S82-9.

2. Graves N, Weinhold D, Tong E, et al. Effect of healthcare-acquired infection on length of hospital stay and cost. Infect Control Hosp Epidemiol. 2007;28:280-92.

3. Raoult D, Richet H. Nosocomial bacteraemia: the most neglected causes of death in Europe? Clin Microbiol Infect. 2011;17(8):1123.

4. Sax H, Allegranzi B, Uçkay I, Larson E, Boyce J, Pittet D. "My five moments for hand hygiene": a user-centred design approach to understand, train, monitor and report hand hygiene. J Hosp Infect. 2007;67:9-21.

5. WHO. WHO guidelines on hand hygiene in health care (advanceddraft): a summary. Clean hands are safer hands. 2005. http://www.who.int/ patientsafety/events/05/HH_en.pdf. Accessed 19 Feb 2008.

6. Polgreen PM, Tassier TL, Pemmaraju SV, Segre AM. Prioritizing healthcare worker vaccinations on the basis of social network analysis. Infect Control Hosp Epidemiol. 2010;31(9):893-900.

7. Temime L, Opatowski L, Pannet Y, Brun-Buisson C, Boëlle P-Y, Guillemot D. Peripatetic health-care workers as potential superspreaders. Proc Natl Acad Sci USA. 2009;106:18420.

8. Boyce JM. Measuring healthcare worker hand hygiene activity: current practices and emerging technologies. Infect Control Hosp Epidemiol. 2011;32:1016.

9. Boudjema S, Dufour JC, Aladro AS, Desquerres I, Brouqui P. MediHandTrace $^{\circledR}$ : a tool for measuring and understanding hand hygiene adherence. Clin Microbiol Infect. 2014;20(1):22-8.
10. Polgreen PM, Hlady CS, Severson MA, Segre AM, Herman T. Method for automated monitoring of hand hygiene adherence without radio-frequency identification. Infect Control Hosp Epidemiol. 2010;31(12):1294-7.

11. SocioPatterns website. http://www.sociopatterns.org/. Accessed $14 \mathrm{Apr}$ 2015.

12. Cattuto C, Van den Broeck W, Barrat A, Colizza V, Pinton J-F, Vespignani A. Dynamics of person-to-person interactions from distributed RFID sensor networks. PLoS One. 2010;5(7):e11596.

13. Stopczynski A, Sekara V, Sapiezynski P, et al. Measuring large-scale social networks with high resolution. PLoS One. 2014;9(4):e95978.

14. Salathé M, Kazandjieva M, Lee JW, et al. A high-resolution human contact network for infectious disease transmission. Proc Natl Acad Sci USA. 2010;107(51):22020-5.

15. Hornbeck T, Naylor D, Segre AM, et al. Using sensor networks to study the effect of peripatetic healthcare workers on the spread of hospitalassociated infections. J Infect Dis. 2012;206:1549.

16. Isella $L$, Romano M, Barrat $A$, et al. Close encounters in a pediatric ward: measuring face-to-face proximity and mixing patterns with wearable sensors. PLoS One. 2011;6(2):e17144.

17. Vanhems P, Barrat A, Cattuto C, et al. Estimating potential infection transmission routes in hospital wards using wearable proximity sensors. PLoS One. 2013;6:e73970

18. Obadia T, Silhol R, Opatowski L, et al. Detailed Contact Data and the Dissemination of Staphylococcus aureus in Hospitals. PLoS Comput Biol. 2015;11(3):e1004170.

19. Voirin N, Payet C, Barrat A, et al. Combining high-resolution contact data with virological data to investigate influenza transmission in a tertiary care hospital. Infect Control Hosp Epidemiol. 2015;36:254.

20. Machens A, Gesualdo F, Rizzo C, Tozzi AE, Barrat A, Cattuto C. An infectious disease model on empirical networks of human contact: bridging the gap between dynamic network data and contact matrices. BMC Infect Dis. 2013;13:185.

21. Srigley JA, Gardam M, Fernie G, Lightfoot D, Lebovic G, Muller MP. Hand hygiene monitoring technology: a systematic review of efficacy. J Hosp Infect. 2015;89(1):51-60.

22. Armellino D, Hussain E, Schilling ME, et al. Using high-technology to enforce low-technology safety measures: the use of third-party remote video auditing and real-time feedback in healthcare. Clin Infect Dis. 2012;54(1):1-7.

\section{Submit your next manuscript to BioMed Central and take full advantage of:}

- Convenient online submission

- Thorough peer review

- No space constraints or color figure charges

- Immediate publication on acceptance

- Inclusion in PubMed, CAS, Scopus and Google Scholar

- Research which is freely available for redistribution

Submit your manuscript at 